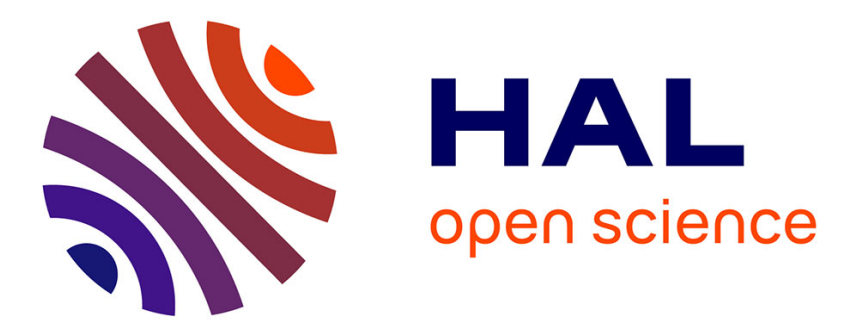

\title{
Tunnel effect in a shrinking shell enlacing a magnetic field
}

\author{
Ayman Kachmar, Nicolas Raymond
}

\section{To cite this version:}

Ayman Kachmar, Nicolas Raymond. Tunnel effect in a shrinking shell enlacing a magnetic field. 2017. hal-01582532v2

\section{HAL Id: hal-01582532 \\ https://hal.science/hal-01582532v2}

Preprint submitted on 9 Sep 2017

HAL is a multi-disciplinary open access archive for the deposit and dissemination of scientific research documents, whether they are published or not. The documents may come from teaching and research institutions in France or abroad, or from public or private research centers.
L'archive ouverte pluridisciplinaire HAL, est destinée au dépôt et à la diffusion de documents scientifiques de niveau recherche, publiés ou non, émanant des établissements d'enseignement et de recherche français ou étrangers, des laboratoires publics ou privés. 


\title{
TUNNEL EFFECT IN A SHRINKING SHELL ENLACING A MAGNETIC FIELD
}

\author{
AYMAN KACHMAR AND NICOLAS RAYMOND
}

\begin{abstract}
Let $C$ be a smooth planar curve. We assume that $C$ is simple, closed, smooth, symmetric with respect to an axis and its curvature attains its minimum at exactly two points away from the axis of symmetry. In a tubular neighborhood about $C$, we study the Laplace operator with a magnetic flux and mixed boundary conditions. As the thickness of the domain tends to 0 , we establish an explicit asymptotic formula for the splitting of the first two eigenvalues (tunneling effect).
\end{abstract}

\section{INTRODUCTION}

1.1. Motivation and context. This paper is devoted to investigate the effect of geometric symmetries on the spectrum of the magnetic Schrödinger operator. This issue is actually quite general: essentially, we want to describe the difference of the first two eigenvalues in some asymptotic limits.

This problem is quite non trivial, especially in the large magnetic field limit. The existing results suggest a tunnel effect when the domain has symmetries (see [1, 3, 7]). However, the formulas for the splitting of the first eigenvalues are still missing, even for two dimensional domains. For ellipses, only a conjecture has been provided in [2] and numerically checked. This conjecture was suggested by a formal dimensional reduction and the analysis in [4, 14].

Some authors have noticed analogies between the magnetic Laplacian and the Robin Laplacian (see for example [8] and [15]). These operators share common features, and, in the Robin case, it has been possible to describe rigorously tunneling effects induced by the geometry, see [9, 10]. The present paper is devoted to the analysis of a hybrid of these two operators. We tackle an elementary geometric situation which can be analyzed via a reduction to dimension one (in the shrinking limit $\varepsilon \rightarrow 0$, which turns out to be of semiclassical nature), in the case when the magnetic field is a pure flux. The behavior of the spectral gap $\lambda_{2}(\varepsilon)-\lambda_{1}(\varepsilon)$ in the limit $\varepsilon \rightarrow 0$ can be established via the methods developed in recent papers (see [4, 9]). Our aim is mainly to explain how these previous contributions can be used to establish a tunneling result involving a pure magnetic field in a limit of semiclassical nature: such results are indeed rather rare in the literature. Since the ingredients have been introduced in our previous works, we will only give the path to the result and highlight the main differences.

\subsection{Framework.}

1.2.1. Geometry. Let $C$ be a simple, smooth and closed curve in $\mathbb{R}^{2}$. The curve $C$ splits $\mathbb{R}^{2}$ into three connected parts

$$
\mathbb{R}^{2}=\Omega \bigcup C \bigcup\left(\mathbb{R}^{2} \backslash \bar{\Omega}\right)
$$

where the set $\Omega$ is an interior domain, i.e. open and bounded.

For $\varepsilon>0$, we define the tubular domain

$$
\Omega_{\varepsilon}=\{x \in \Omega: 0<\operatorname{dist}(x, C)<\varepsilon\}
$$

with "thickness" $\varepsilon$ (see Figure 1). 


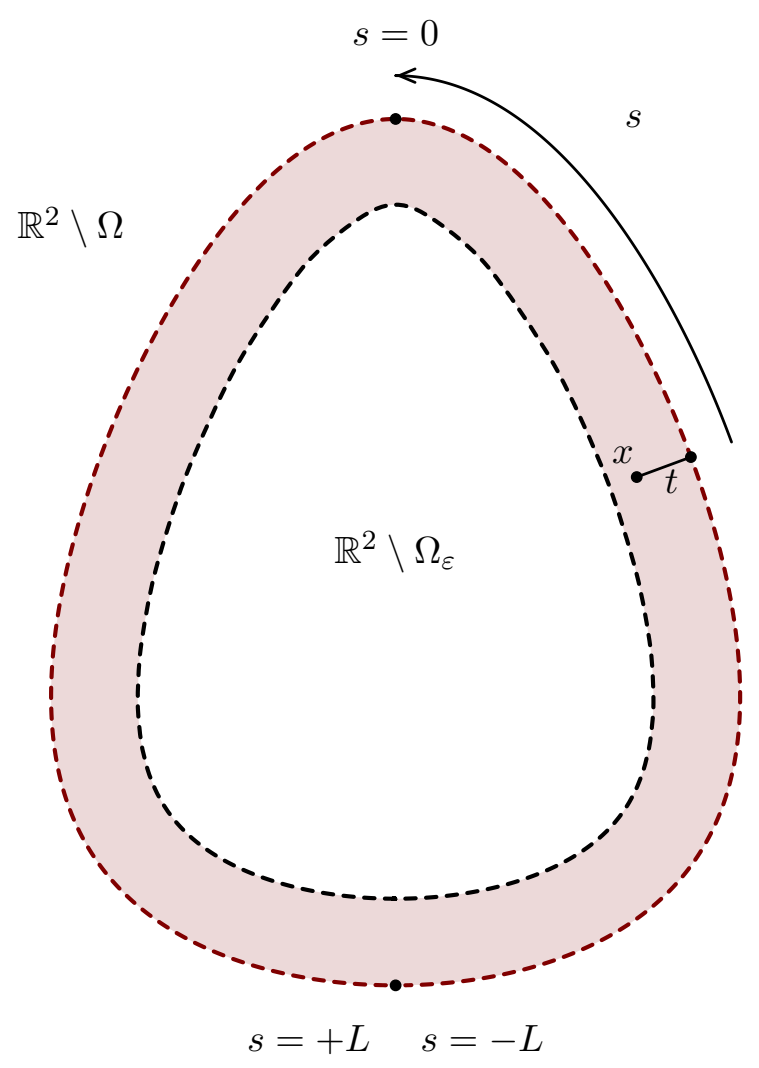

FiguRE 1. The red region is $\Omega_{\varepsilon}$. The dashed red curve carries the Dirichlet condition and the black one the Neumann condition.

1.2.2. Definition of the operator. Let $\mathbf{A}: \mathbb{R}^{2} \rightarrow \mathbb{R}^{2}$ be a vector field satisfying

$$
\operatorname{curl} \mathbf{A}=0 \text { in } \mathbb{R}^{2} \backslash K,
$$

where $K \subset \Omega$ is a compact set. For $\varepsilon>0$, consider the Laplace operator with magnetic field $\mathbf{A}$

$$
\mathcal{L}_{\varepsilon}=-(\nabla-i \mathbf{A})^{2} \quad \text { in } L^{2}\left(\Omega_{\varepsilon}\right),
$$

with Dirichlet boundary condition on $C$ and Neumann boundary condition on $\{\operatorname{dist}(x, C)=\varepsilon\}$.

The analysis of this operator (without a magnetic field) is the subject of the paper [12] in a non-periodic framework. The operator with Dirichlet boundary condition has been studied in numerous papers (see [5, 13 and the references therein). In this setting, the effective operator is independent of $\varepsilon$ and thus does not lead to an asymptotic spectral confinement, unlike the case of mixed Dirichlet-Neumann conditions that we treat here.

Note that when $\varepsilon$ is sufficiently small, curl $\mathbf{A}=0$ in $\Omega_{\varepsilon}$. Consequently, the spectrum of the operator $\mathcal{L}_{\varepsilon}$ depends on $\mathbf{A}$ through the magnetic flux

$$
\mathrm{f}_{0}=\frac{1}{|\partial \Omega|} \int_{\Omega} \operatorname{curl} \mathbf{A} d x
$$

We denote by $\left(\lambda_{n}(\varepsilon)=\lambda_{n}\left(\varepsilon, \mathrm{f}_{0}\right)\right)_{n \geq 1}$ the non-decreasing sequence of eigenvalues of $\mathcal{L}_{\varepsilon}$.

1.3. The effective operator. Let $[-L, L) \ni s \mapsto M(s) \in C$ be the arc-length parameterization of the curve $C$ (see Figure 1). Here the arc-length measure of $C$ is $2 L$. For all $s \in[-L, L$, let $\kappa(s)$ denote the curvature of $C$ at the point $M(s)$. We define the operator

$$
\mathcal{L}_{\varepsilon}^{\mathrm{eff}}=-\left(\frac{d}{d s}-i \mathrm{f}_{0}\right)^{2}+\frac{\kappa(s)}{\varepsilon} \quad \text { in } L^{2}([-L, L)),
$$


with periodic conditions at $\pm L$. Here $\mathrm{f}_{0}$ is the magnetic flux introduced in 1.5 . We will refer to the operator $\mathcal{L}_{\varepsilon}^{\text {eff }}$ as the "effective" operator. As consequence of the analysis in [12], if $\mathrm{f}_{0}=0$ then, for all $n \in \mathbb{N}^{*}$,

$$
\lambda_{n}(\varepsilon)-\left(\frac{\pi}{2 \varepsilon}\right)^{2}=\mu_{n}^{\mathrm{eff}}(\varepsilon)+\mathcal{O}(1)
$$

as $\varepsilon \rightarrow 0_{+}$. This result continues to hold when $\mathrm{f}_{0} \neq 0$ by a slight adjustement of the argument given in [12]. If, moreover, the curvature function $\kappa$ has a unique non-degenerate minimum at $s_{0}$, then the harmonic approximation yields

$$
\mu_{n}^{\mathrm{eff}}(\varepsilon)-\frac{\kappa\left(s_{0}\right)}{\varepsilon} \sim(2 n-1) \sqrt{\frac{\kappa^{\prime \prime}\left(s_{0}\right)}{2}} \frac{1}{\sqrt{\varepsilon}} \quad \text { as } \varepsilon \rightarrow 0_{+} .
$$

Remark 1.1. If we exchange the Dirichlet and Neumann conditions, then $\kappa$ has to be changed into $-\kappa$ and thus we have to consider a point of maximal curvature.

On the contrary, if the function $\kappa$ is even and has two non-degenerate minima, then tunneling occurs and the spectral gap $\mu_{2}^{\text {eff }}(\varepsilon)-\mu_{1}^{\text {eff }}(\varepsilon)$ is exponentially small as $\varepsilon \rightarrow 0_{+}$(see Eq. 1.9p) below). We introduce the electric potential $\mathfrak{v}=\kappa-\kappa_{\text {min }}$ and the following quantities

$$
\mathrm{S}=\min \left(\mathrm{S}_{\mathrm{u}}, \mathrm{S}_{\mathrm{d}}\right), \quad \mathrm{S}_{\mathrm{u}}=\int_{\left[s_{\mathrm{r}}, s_{\ell}\right]} \sqrt{\mathfrak{v}(s)} d s, \quad \mathrm{~S}_{\mathrm{d}}=\int_{\left[s_{\ell}, \mathrm{s}_{\mathrm{r}}\right]} \sqrt{\mathfrak{v}(s)} d s,
$$

where $[p, q]$ denotes the arc joining $p$ and $q$ in $C$ counter-clockwise. The indices $\mathrm{u}$ and $\mathrm{d}$ refer to the up and down parts of $C$. Using a rescaling and [4, Theorem 1.4] (see also [14]), we have

$$
\mu_{2}^{\mathrm{eff}}(\varepsilon)-\mu_{1}^{\mathrm{eff}}(\varepsilon)=2|w(\varepsilon)|+\mathcal{O}\left(\varepsilon^{-\frac{1}{4}} e^{-\mathrm{S} / \varepsilon^{1 / 2}}\right),
$$

where

$$
w(\varepsilon)=2 \varepsilon^{-\frac{3}{4}} \pi^{-\frac{1}{2}} \gamma^{\frac{1}{2}}\left(\mathrm{~A}_{\mathrm{u}} \sqrt{\mathfrak{v}(0)} e^{-\mathrm{S}_{\mathrm{u}} / \varepsilon^{1 / 2}} e^{-i L \mathrm{f}_{0}}+\mathrm{A}_{\mathbf{d}} \sqrt{\mathfrak{v}(L)} e^{-\mathrm{S}_{\mathrm{d}} / \varepsilon^{1 / 2}} e^{i L \mathrm{f}_{0}}\right),
$$

with

$$
\begin{aligned}
& \mathrm{A}_{\mathrm{u}}=\exp \left(-\int_{\left[s_{\mathrm{r}}, 0\right]} \frac{\left(\mathfrak{v}^{\frac{1}{2}}\right)^{\prime}(s)+\gamma}{\sqrt{\mathfrak{v}(s)}} d s\right), \\
& \mathrm{A}_{\mathrm{d}}=\exp \left(-\int_{\left[s_{\ell}, L\right]} \frac{\left(\mathfrak{v}^{\frac{1}{2}}\right)^{\prime}(s)-\gamma}{\sqrt{\mathfrak{v}(s)}} d s\right), \\
& \gamma=\left(\mathfrak{v}^{\prime \prime}\left(s_{\mathrm{r}}\right) / 2\right)^{\frac{1}{2}}=\left(\mathfrak{v}^{\prime \prime}\left(s_{\ell}\right) / 2\right)^{\frac{1}{2}} .
\end{aligned}
$$

and $f_{0}$ is the flux introduced in 1.5 .

1.4. Main result. In the symmetric case of Assumption 1.2 below, the formula in (1.7) for the two dimensional setting is not enough to describe exponentially small effects. We will improve it in this special case.

In the rest of this paper, we will assume that the curve $C$ is symmetric about the $y$-axis and the curvature has two non-degenerate minima. In terms of the arc-length parametrization $[-L, L) \ni s \mapsto M(s)=(x(s), y(s))$ of $C$, we reformulate our assumption as follows (see also Figure 1).

\section{Assumption 1.2.}

i. For all $s \in(-L, L), M(s)=(x(s), y(s))=(x(-s),-y(s))$,

ii. The function $s \mapsto \kappa(s)$ is even and admits two non-degenerate minima at $s_{l} \in(0, L)$ and $s_{r} \in(-L, 0)$, such that

$$
\kappa^{\prime \prime}\left(s_{\ell}\right)=\kappa^{\prime \prime}\left(s_{r}\right)=k_{2}>0 .
$$

Figure 1 illustrates Assumption 1.2. Our result is the following theorem. 
Theorem 1.3. Under Assumption 1.2, the spectral gap satisfies

$$
\lambda_{2}(\varepsilon)-\lambda_{1}(\varepsilon) \sim \mu_{2}^{\mathrm{eff}}(\varepsilon)-\mu_{1}^{\mathrm{eff}}(\varepsilon) \text { as } \varepsilon \rightarrow 0_{+} .
$$

Moreover, the asymptotic behavior of $\mu_{2}^{\mathrm{eff}}(\varepsilon)-\mu_{1}^{\mathrm{eff}}(\varepsilon)$ is described in 1.9 ).

Remark 1.4. As noticed in Remark 1.1, if we exchange the Dirichlet and Neumann conditions, the points of maximal curvature play a crucial role. In this case, if there are two symmetric points of (non-degenerate) maximal curvature, the same kind of tunneling formula is true.

1.5. Organization of the paper. The paper is organized as follows. In Section 2 , we describe the operators involved in the proof of the main theorem. In Section 3, we explain why (and in which sense) the first two eigenfunctions of $\mathcal{L}_{\varepsilon}$ are localized near the points of minimal curvature. Section 4 is devoted to the WKB approximation of the ground states for the one well problems. In Section 5, we describe the interaction between the wells.

\section{Operators}

2.1. The transversal operators. In this section, we discuss the spectral properties of one dimensional operators which are involved in the proof of the main result (Theorem 1.3).

2.1.1. The free operator. Consider the operator $\mathcal{L}_{0}^{1 D}=-\frac{d^{2}}{d \tau^{2}}$ in $L^{2}(0,1)$ with Dirichlet condition at $\tau=0$ and Neumann condition at $\tau=1$. The spectrum of this operator consists of the simple eigenvalues

$$
\lambda_{n}^{1 D}=\left(\frac{\pi}{2}+(n-1) \pi\right)^{2} \quad(n=1,2, \cdots) .
$$

The $L^{2}$-normalized ground state of this operator is

$$
u_{0}(\tau)=\sqrt{2} \sin \left(\frac{\pi \tau}{2}\right) .
$$

2.1.2. The weighted operator. Let $\beta \in(0,1)$. Consider the operator

$$
\mathcal{L}_{\beta}^{1 D}=-(1-\beta \tau)^{-1} \frac{d}{d \tau}\left((1-\beta \tau) \frac{d}{d \tau}\right)
$$

acting on the weighted space $L^{2}((0,1) ;(1-\beta \tau) d \tau)$ with Dirichlet condition at $\tau=0$ and Neumann condition at $\tau=1$.

Let $\left(\lambda_{n}^{1 D}(\beta)\right)_{n \geq 1}$ be the sequence of eigenvalues of the operator $\mathcal{L}_{\beta}^{1 D}$. Arguing as in 8 , Lem. 4.4\&Prop 4.5] (see also [12]), there exist $C>0$ and $\beta_{0} \in(0,1)$ such that, for all $\beta \in\left(0, \beta_{0}\right)$, we have

$$
\left|\lambda_{1}^{1 D}(\beta)-\left(\frac{\pi}{2}\right)^{2}+\beta\right| \leq C \beta^{2}, \quad \lambda_{2}^{1 D}(\beta) \geq\left(\frac{3 \pi}{2}\right)^{2}-C \beta .
$$

\subsection{The tubular operator.}

2.2.1. The tubular coordinates. We will use the canonical tubular coordinates $(s, t)$ where $s$ is the arc-length and $t$ is the distance to the boundary (see Figure 1). We will describe these coordinates here. Recall that

$$
[-L, L) \ni s \mapsto M(s) \in \Gamma
$$

is the arc-length parametrization of the curve $C$. The unit tangent vector of $C$ at the point $M(s)$ of the boundary is given by

$$
T(s)=M^{\prime}(s) .
$$

We define the curvature $\kappa(s)$ by the following identity

$$
T^{\prime}(s)=\kappa(s) \nu(s),
$$


where $\nu(s)$ is the unit vector, normal to the curve $C$, pointing outward at the point $M(s)$. We choose the orientation of the parametrization $M$ to be counter-clockwise, so that, for all $s \in[-L, L)$,

$$
\operatorname{det}(T(s), \nu(s))=1
$$

We introduce the change of coordinates

$$
\Phi:(-L, L] \times(0, \varepsilon) \ni(s, t) \mapsto x=M(s)-t \nu(s) \in \Omega_{\varepsilon} .
$$

The determinant of the Jacobian of $\Phi$ is given by

$$
a(s, t)=1-t \kappa(s) .
$$

In light of Assumption 1.2 , the choice of the origin of the parametrization is the point $M(0)$ such that $[M(-L), M(0)]$ defines the axis of symmetry. This is illustrated in Figure 1 .

2.2.2. The operator in the new coordinates. We will express the operator $\mathcal{L}_{\varepsilon}$ in the $(s, t)$ coordinates. For all $u \in L^{2}\left(\Omega_{\varepsilon}\right)$, we define the pull-back function

$$
\widetilde{u}(s, t)=u(\Phi(s, t)) .
$$

The magnetic potential A induces a new magnetic potential $\widetilde{\mathbf{A}}$ in the $(s, t)$ coordinates. The components of $\widetilde{\mathbf{A}}$ are obtained by projecting the vector field $\mathbf{A}$ on the normal and tangential vectors. Applying a gauge transformation (in the $(s, t)$ coordinates), we may assume that $\widetilde{\mathbf{A}}$ satisfies (see [6, Proof of Lem. F.1.1])

$$
\widetilde{\mathbf{A}}(s, t)=\left(\mathrm{f}_{0}, 0\right),
$$

where $\mathrm{f}_{0}$ is the magnetic flux introduced in 1.5 .

In this way, we see that the operator $\mathcal{L}_{\varepsilon}$ is unitary equivalent to the operator (see [6, Eq. (F.4)(F.5)])

$$
\widetilde{\mathcal{L}}_{\varepsilon}=-a^{-1}\left(\partial_{s}-i \mathrm{f}_{0}\right)\left(a^{-1}\left(\partial_{s}-i \mathrm{f}_{0}\right)\right)-a^{-1} \partial_{t}\left(a \partial_{t}\right) \text { in } L^{2}([-L, L) \times(0, \varepsilon) ; a d s d t),
$$

where $a$ is introduced in 2.5 . The boundary conditions for $\widetilde{\mathcal{L}}_{\varepsilon}$ are as follows: periodic boundary conditions at $s= \pm L$, Dirichlet condition at $t=0$ and Neumann condition at $t=\varepsilon$.

Performing the rescaling $t=\varepsilon \tau$ and multiplying by $\varepsilon^{2}$ (to have a "semiclassical normalization"), we obtain the new operator

$$
\mathcal{P}_{\varepsilon}=-\varepsilon^{2} a_{\varepsilon}^{-1}\left(\partial_{s}-i \mathrm{f}_{0}\right)\left(a_{\varepsilon}^{-1}\left(\partial_{s}-i \mathrm{f}_{0}\right)\right)-a_{\varepsilon}^{-1} \partial_{\tau}\left(a_{\varepsilon} \partial_{\tau}\right) \text { in } L^{2}\left([-L, L) \times(0,1) ; a_{\varepsilon} d s d t\right),
$$

where

$$
a_{\varepsilon}(s, \tau)=1-\varepsilon \kappa(s) \tau .
$$

The quadratic form of the operator $\mathcal{P}_{\varepsilon}$ is

$$
q_{\varepsilon}(v)=\int_{-L}^{L} \int_{0}^{1}\left(\varepsilon^{2} a_{\varepsilon}^{-2}\left|\left(\partial_{s}-i \mathrm{f}_{0}\right) v\right|^{2}+\left|\partial_{\tau} v\right|^{2}\right) a_{\varepsilon}(s, \tau) d \tau d s,
$$

defined on the form domain

$$
\operatorname{Dom}\left(q_{\varepsilon}\right)=\left\{v \in H^{1}((-L, L) \times(0,1)): v(0)=0, \quad v(L)=v(-L)\right\} .
$$

Note that the $n$-th eigenvalue, $\lambda_{n}(\varepsilon)$, of $\mathcal{L}_{\varepsilon}$ is given now as

$$
\lambda_{n}(\varepsilon)=\varepsilon^{-2} \lambda_{n}\left(\mathcal{P}_{\varepsilon}\right) .
$$




\section{Agmon estimates in the double well CASe}

3.1. Lower bound of the quadratic form and consequences. Using (2.11), (2.2) with $\beta=\varepsilon \kappa(s)$, and the min-max principle, we obtain the following inequality

$$
q_{\varepsilon}(v) \geq \int_{-L}^{L} \int_{0}^{1}\left(\varepsilon^{2} a_{\varepsilon}^{-2}\left|\left(\partial_{s}-i \mathrm{f}_{0}\right) v\right|^{2}+\left(\frac{\pi}{2}\right)^{2}+\left(\varepsilon \kappa(s)-\mathcal{O}\left(\varepsilon^{2}\right)\right)|v|^{2}\right) a_{\varepsilon}(s, t) d s d \tau,
$$

where $a_{\varepsilon}$ is introduced in $(2.10)$. Thus, we recognize the quadratic of the effective operator (see (1.6)) in this lower bound.

Using (3.1) and classical estimates for one dimensional electric Schrödinger operators, we can derive the localization of the first eigenfunctions of $\mathcal{P}_{\varepsilon}$ near the points of minimal curvature $s_{\ell}$ and $s_{r}$. The proof of the following proposition is a straightforward adaptation of the one of [9, Prop. 4.7].

Proposition 3.1. Let $\alpha \in(0,1)$. There exist constants $C, \varepsilon_{0}>0$ such that, for all $\varepsilon \in\left(0, \varepsilon_{0}\right)$ and all $u_{\varepsilon} \in \bigoplus_{i=1}^{2} \operatorname{Ker}\left(\mathcal{P}_{\varepsilon}-\lambda_{i}\left(\mathcal{P}_{\varepsilon}\right)\right)$,

$$
\int_{0}^{1} \int_{-L}^{L} e^{2 \Phi_{\alpha} / \sqrt{\varepsilon}}\left(\left|\left(\partial_{s}-i \mathrm{f}_{0}\right) u_{\varepsilon}\right|^{2}+\left|u_{\varepsilon}\right|^{2}\right) a_{\varepsilon}(s, \tau) d s d \tau \leq C \varepsilon^{1 / 2}\left\|u_{\varepsilon}\right\|^{2}
$$

where

$$
\Phi_{\alpha}=\sqrt{1-\alpha} \Phi, \quad \Phi=\min \left(\Phi_{r}, \Phi_{l}\right),
$$

with

and

$$
\forall \sigma \in[-L, L), \quad \Phi_{r}(s)=\int_{\left[s_{r}, s\right]} \sqrt{\kappa(\sigma)-\kappa_{\min }} d \sigma
$$

$$
\forall \sigma \in[-L, L), \quad \Phi_{\ell}(s)=\int_{\left[s_{\ell}, s\right]} \sqrt{\kappa(\sigma)-\kappa_{\min }} d \sigma .
$$

Notation 3.2. For $j \in\{r, \ell\}$, the integral $\int_{\left[s_{j}, s\right]} f(\sigma) d \sigma$ in Proposition 3.1 is understood as the (connected) line integral on the curve $C \backslash\left\{s_{\bar{j}}\right\}$ between the points $s_{j}$ and $s$ in the counter clock-wise direction.

Proposition 3.1 can be generalized as follows (cf. [9, Prop 6.1]).

Proposition 3.3. For all $\alpha \in(0,1)$ and $C_{0}>0$, there exist positive constants $\varepsilon_{0}, A, c, C$ such that, for all $\varepsilon \in\left(0, \varepsilon_{0}\right), z \in\left[\left(\frac{\pi}{2}\right)^{2}+\kappa_{\min } \varepsilon,\left(\frac{\pi}{2}\right)^{2}+\kappa_{\min } \varepsilon+C_{0} \varepsilon^{3 / 2}\right]$ and $u \in \operatorname{Dom}\left(\mathcal{P}_{\varepsilon}\right)$, the following inequalities hold,

$$
c \varepsilon^{3 / 2}\left\|e^{\Phi_{\alpha} / \sqrt{\varepsilon}} u\right\|_{L^{2}} \leq\left\|e^{\Phi_{\alpha} / \sqrt{\varepsilon}}\left(\mathcal{P}_{\varepsilon}-z\right) u\right\|_{L^{2}}+C \varepsilon^{3 / 2}\|u\|_{L^{2}\left(\widehat{\mathcal{B}}\left(A \varepsilon^{\frac{1}{4}}\right)\right)},
$$

and

$$
\varepsilon^{2}\left\|\partial_{\sigma}\left(e^{-i \mathrm{f}_{0} s} e^{\Phi_{\alpha} / \sqrt{\varepsilon}} u\right)\right\|_{L^{2}}^{2} \leq C \varepsilon^{-3 / 2}\left\|e^{\Phi_{\alpha} / \sqrt{\varepsilon}}\left(\mathcal{P}_{\varepsilon}-z\right) u\right\|_{L^{2}}^{2}+C \varepsilon^{3 / 2}\|u\|_{L^{2}\left(\widehat{\mathcal{B}}\left(A \varepsilon^{\frac{1}{4}}\right)\right)}^{2},
$$

where $\widehat{\mathcal{B}}(\varrho)=\left\{s \in[-L, L):\left|s-s_{\ell}\right| \geq \rho\right.$ and $\left.\left|s-s_{r}\right| \geq \rho\right\}$.

3.2. Rough localization of the spectrum. We recall that $\mathcal{P}_{\varepsilon}$ is the operator introduced in (2.9). Thanks to Proposition 3.1, we deduce that, in order to estimate the first eigenvalues, the two wells can be decoupled modulo an exponentially small remainder. Then, using (1.7) and the known results about the effective operator in (1.6), we get the following proposition.

Proposition 3.4. There exist $\varepsilon_{0}, c_{0}>0$ such that, for all $\varepsilon \in\left(0, \varepsilon_{0}\right)$,

$$
\begin{aligned}
& \operatorname{Spec}\left(\mathcal{P}_{\varepsilon}\right) \cap\left[\left(\frac{\pi}{2}\right)^{2}+\varepsilon \kappa_{\min }+\varepsilon^{3 / 2} \sqrt{\frac{k_{2}}{2}}-c_{0} \varepsilon^{2},\left(\frac{\pi}{2}\right)^{2}+\varepsilon \kappa_{\min }+\varepsilon^{3 / 2} \sqrt{\frac{k_{2}}{2}}+c_{0} \varepsilon^{2}\right] \\
&=\left\{\lambda_{1}\left(\mathcal{P}_{\varepsilon}\right), \lambda_{2}\left(\mathcal{P}_{\varepsilon}\right)\right\}
\end{aligned}
$$


and

$$
\lambda_{3}\left(\mathcal{P}_{\varepsilon}\right) \geq\left(\frac{\pi}{2}\right)^{2}+\varepsilon \kappa_{\min }+3 \varepsilon^{3 / 2} \sqrt{\frac{k_{2}}{2}}-c_{0} \varepsilon^{2},
$$

where $k_{2}$ is the constant introduced in Assumption 1.2 and $\kappa_{\min }=\min _{s \in[-L, L)} \kappa(s)$.

The next sections are devoted to estimate the (exponentially small) difference $\lambda_{2}\left(\mathcal{P}_{\varepsilon}\right)-\lambda_{1}\left(\mathcal{P}_{\varepsilon}\right)$.

\section{The one Well operators}

4.1. Definition of the operators. We introduce the geometric constant (see Assumption 1.2 )

$$
\eta^{*}=\min \left(\frac{L}{2},\left|s_{r}+L\right|,\left|s_{r}\right|\right) .
$$

Let $\eta \in\left(0, \eta^{*}\right)$ be a given constant. Define the "right" well operator

$$
\widetilde{\mathcal{P}}_{\varepsilon, r}=-\varepsilon^{2} a_{\varepsilon}^{-1}\left(\partial_{s}-i \mathrm{f}_{0}\right)\left(a_{\varepsilon}^{-1}\left(\partial_{s}-i \mathrm{f}_{0}\right)\right)-a_{\varepsilon}^{-1} \partial_{\tau}\left(a_{\varepsilon} \partial_{\tau}\right) \text { in } L^{2}\left(U_{\eta, r} ; a_{\varepsilon} d s d t\right),
$$

where

$$
U_{\eta, r}=I_{\eta, r} \times(0,1), \quad I_{\eta, r}=\left(s_{\ell}+\eta-2 L, s_{\ell}-\eta\right) .
$$

We assume that the functions in the domain of $\widetilde{\mathcal{P}}_{\varepsilon, r}$ satisfy the Neumann boundary condition at $t=1$ and the Dirichlet condition elsewhere. When $\mathrm{f}_{0}=0$, we will write $\widetilde{\mathcal{P}}_{\varepsilon, r}=\mathfrak{P}_{\varepsilon, r}$. Due to the periodicity, the operator $\widetilde{\mathcal{P}}_{\varepsilon, r}$ is unitarily equivalent to the operator $\mathcal{P}_{\varepsilon, r}$ acting as

$$
-\varepsilon^{2} a_{\varepsilon}^{-1}\left(\partial_{s}-i \mathrm{f}_{0}\right)\left(a_{\varepsilon}^{-1}\left(\partial_{s}-i \mathrm{f}_{0}\right)\right)-a_{\varepsilon}^{-1} \partial_{\tau}\left(a_{\varepsilon} \partial_{\tau}\right),
$$

on $L^{2}\left(\left(-L, s_{\ell}-\eta\right) \cup\left(s_{\ell}+\eta, L\right) ; a_{\varepsilon} d s d t\right)$ and subject to the periodic condition at $\pm L$, the Neumann boundary condition at $\tau=1$ and the Dirichlet condition elsewhere.

Similarly, we define the "left" well operator

$$
\widetilde{\mathcal{P}}_{\varepsilon, l}=-\varepsilon^{2} a_{\varepsilon}^{-1}\left(\partial_{s}-i \mathrm{f}_{0}\right)\left(a_{\varepsilon}^{-1}\left(\partial_{s}-i \mathrm{f}_{0}\right)\right)-a_{\varepsilon}^{-1} \partial_{\tau}\left(a_{\varepsilon} \partial_{\tau}\right) \text { in } L^{2}\left(U_{\eta, l} ; a_{\varepsilon} d s d t\right),
$$

where

$$
U_{\eta, l}=I_{\eta, \ell} \times(0,1), \quad I_{\eta, \ell}=\left(s_{r}+\eta, s_{r}-\eta+2 L\right) .
$$

We assume that the functions in the domain of $\widetilde{\mathcal{P}}_{\varepsilon, \ell}$ satisfy the Neumann boundary condition at $t=1$ and the Dirichlet condition elsewhere. We also consider the flux free version $\mathfrak{P}_{\varepsilon, \ell}$, and $\mathcal{P}_{\varepsilon, \ell}$ the realization of the operator on $\left(s_{r}+\eta, L\right) \cup\left(-L, s_{r}-\eta+2 L\right)$ with periodic condition on $s= \pm L$.

With Assumption 1.2, we are led to introduce the unitary transform

$$
U f(s, t)=\overline{f(-s, t)},
$$

and we notice that $\left[\mathcal{P}_{\varepsilon}, U\right]=0$. Moreover, we have $U^{-1} \mathcal{P}_{\varepsilon, r} U=\mathcal{P}_{\varepsilon, l}$. Thus, the operators $\mathcal{P}_{\varepsilon, r}$ and $\mathcal{P}_{\varepsilon, l}$ are unitary equivalent. Consequently, we denote by $\mu_{1}^{\mathrm{sw}}(\varepsilon)$ the first eigenvalue of the operators $\mathcal{P}_{\varepsilon, r}$ and $\mathcal{P}_{\varepsilon, l}$. We suppressed $\eta$ from the notation of $\mu_{1}^{\mathrm{sw}}(\varepsilon)$ for the sake of simplicity, and because the dependence on $\eta$ is unimportant in our analysis.

We define the function $\phi_{\varepsilon, r}$ by

$$
\phi_{\varepsilon, r}(s, t)=\left\{\begin{array}{ll}
e^{-i \mathrm{f}_{0} s} u_{\varepsilon, r}(s, t) & \text { if }-L \leq s \leq s_{l}-\eta \\
e^{-i \mathrm{f}_{0}(s-2 L)} u_{\varepsilon, r}(s-2 L, t) & \text { if } s_{l}+\eta \leq s<L
\end{array} .\right.
$$

Here $u_{\varepsilon, r}$ is the positive $L^{2}$-normalized ground state of $\mathfrak{P}_{\varepsilon, r}$, the operator without magnetic flux.

The function $\phi_{\varepsilon, r}$ is a ground state of $\mathcal{P}_{\varepsilon, r}$. We let $\phi_{\varepsilon, l}=U \phi_{\varepsilon, r}$. It is a ground state of $\mathcal{P}_{\varepsilon, l}$. Recalling that $s_{l}=-s_{r}$, we have explicitly

$$
\phi_{\varepsilon, l}(s, t)=\left\{\begin{array}{ll}
e^{-i \mathrm{f}_{0}(s+2 L)} u_{\varepsilon, l}(s+2 L, t) & \text { if }-L \leq s \leq s_{r}-\eta \\
e^{-i \mathrm{f}_{0} s} u_{\varepsilon, l}(s, t) & \text { if } s_{r}+\eta \leq s<L
\end{array},\right.
$$

where $u_{\varepsilon, l}$ is the positive $L^{2}$-normalized ground state of the Laplace operator $\mathfrak{P}_{\varepsilon, \ell}$ in $U_{\eta, l}$. Note that $u_{\varepsilon, l}=U u_{\varepsilon, r}$. 
Remark 4.1. In (4.7), we have to change the gauge factor in order to satisfy the periodic boundary condition on $s= \pm L$.

In the sequel, we will analyze the ground state $\phi_{\varepsilon, r}$, or equivalently $u_{\varepsilon, r}$. The properties of $\phi_{\varepsilon, r}$ will be mapped to $\phi_{\varepsilon, l}$ via the symmetry relation.

As for the second eigenvalue of the operators $\mathcal{P}_{\varepsilon, r}$ and $\mathcal{P}_{\varepsilon, \ell}$, it satisfies (see [12]):

$$
\mu_{2}^{\mathrm{sw}}(\varepsilon)=\left(\frac{\pi}{2}\right)^{2}+\varepsilon \kappa_{\min }+3 \sqrt{\frac{k_{2}}{2}} \varepsilon^{3 / 2}+\mathcal{O}\left(\varepsilon^{2}\right) \quad \text { as } \varepsilon \rightarrow 0_{+} .
$$

4.2. Agmon estimates. Similarly as Proposition 3.1, the concentration of the ground state $\phi_{\varepsilon, r}$ near the point $s_{r}$ can be quantified by an Agmon type estimate (see [9, Prop. 4.7] for the proof).

Proposition 4.2. Let $\alpha \in(0,1)$. There exist constants $C, \varepsilon_{0}>0$ such that, for all $\varepsilon \in\left(0, \varepsilon_{0}\right)$,

$$
\int_{\left(-L, s_{\ell}-\eta\right) \cup\left(s_{\ell}+\eta, L\right)} e^{2 \Phi_{\alpha, r} / \sqrt{\varepsilon}}\left(\left|\left(\partial_{s}-i \mathrm{f}_{0}\right) \phi_{\varepsilon, r}\right|^{2}+\left|\phi_{\varepsilon, r}\right|^{2}\right) a_{\varepsilon}(s, \tau) d s d \tau \leq C \varepsilon^{1 / 2}\left\|\phi_{\varepsilon, r}\right\|^{2},
$$

where

$$
\Phi_{\alpha, r}(s)=\sqrt{1-\alpha} \int_{\left[s_{r}, s\right]} \sqrt{\kappa(\sigma)-\kappa_{\min }} d \sigma .
$$

4.3. The WKB construction. Let us now describe the WKB approximation of $u_{\varepsilon, r}$, the ground state of the Laplace operator $\mathfrak{P}_{\epsilon, r}$ in $U_{\eta, r}$ (see 4.3).

Proposition 4.3. There exist a sequence of smooth functions $\left(a_{j}\right)_{j \geq 0}$ and a sequence of real numbers $\left(\mu_{j}\right)_{j \geq 3}$ such that the following holds. The function defined via the formal series

$$
\Psi_{\varepsilon, r}(s, \tau) \sim \varepsilon^{-\frac{1}{4}} e^{-\Phi_{r}(s) / \sqrt{\varepsilon}} \sum_{j \geq 0} \varepsilon^{\frac{j}{2}} a_{j}(s, \tau),
$$

satisfies

$$
e^{\Phi_{r} / \sqrt{\varepsilon}}\left(\mathfrak{P}_{\varepsilon, r}-\mu\right) \Psi_{\varepsilon, r}=\mathcal{O}\left(\varepsilon^{\infty}\right),
$$

where $\mu$ is an asymptotic series in the form

$$
\mu \sim\left(\frac{\pi}{2}\right)^{2}+\varepsilon \kappa_{\min }+\varepsilon^{3 / 2} \sqrt{\frac{k_{2}}{2}}+\sum_{j \geq 3} \mu_{j} \varepsilon^{j / 2},
$$

and

\section{Furthermore}

$$
\Phi_{r}(s)=\int_{\left[s_{r}, s\right]} \sqrt{\kappa(\sigma)-\kappa_{\min }} d \sigma
$$

i) $a_{0}$ is in the form $a_{0}(s, \tau)=\xi_{0, r}(\sigma) u_{0}(\tau)$ where

and

$$
u_{0}(\tau)=\sqrt{2} \sin \left(\frac{\pi \tau}{2}\right)
$$

$$
\xi_{0, r}(s)=\xi_{0}(s)=\left(\frac{\gamma}{\pi}\right)^{\frac{1}{4}} \exp \left(-\int_{s_{r}}^{s} \frac{\Phi_{r}^{\prime \prime}-\gamma}{2 \Phi_{r}^{\prime}} d \sigma\right)
$$

is the solution of the transport equation of the effective Hamiltonian

$$
\Phi_{r}^{\prime} \partial_{s} \xi_{0}+\partial_{s}\left(\Phi_{r}^{\prime} \xi_{0}\right)=\sqrt{\frac{k_{2}}{2}} \xi_{0} \quad \text { with } \gamma=\sqrt{\frac{k_{2}}{2}} \text { and } k_{2} \text { given in } 1.10 \text {. }
$$

ii) For $j \geq 1, a_{j}(\sigma, \tau)$ is a linear combination of smooth functions

$$
f_{j, k}(\sigma) g_{j, k}(\tau)
$$

and satisfy the Dirichlet condition at $\tau=0$, and Neumann condition at $\tau=1$. 
Proof. We expand the operator $\mathfrak{P}_{\varepsilon, r}$ formally as follows

$$
\begin{aligned}
\mathfrak{P}_{\varepsilon, r} \sim-\partial_{\tau}^{2}- & \varepsilon^{2} \partial_{s}^{2}+2 \varepsilon^{3} \tau \kappa(\sigma) \partial_{s}^{2}+\varepsilon \kappa(\sigma) \partial_{\tau}+\varepsilon^{3} \tau \kappa^{\prime}(s) \partial_{s} \\
& -\sum_{j=1}^{\infty} c_{j} \varepsilon^{j+2} \tau^{j}(\kappa(s))^{j} \partial_{s}^{2}+\sum_{j=1}^{\infty} \varepsilon^{j+1} \tau^{j}(\kappa(s))^{j+1} \partial_{\tau}-\kappa^{\prime}(s) \sum_{j=1}^{\infty} \varepsilon^{j+3} d_{j} \tau^{j}(\kappa(s))^{j} \partial_{s} .
\end{aligned}
$$

We introduce the (formal) conjugate operator

$$
\mathfrak{P}_{\varepsilon, r}^{\vartheta}=\exp \left(\frac{\vartheta(s)}{\sqrt{\varepsilon}}\right) \mathcal{P}_{\varepsilon, r} \exp \left(-\frac{\vartheta(s)}{\sqrt{\varepsilon}}\right)
$$

and expand it formally as follows

$$
\mathfrak{P}_{\varepsilon, r}^{\vartheta} \sim \sum_{\ell \geq 0} Q_{\ell}^{\vartheta} \varepsilon^{\ell / 2}
$$

with

$$
\begin{aligned}
& Q_{0}^{\vartheta}=-\partial_{\tau}^{2}, \\
& Q_{1}^{\vartheta}=0 \\
& Q_{2}^{\vartheta}=\kappa(s) \partial_{\tau}-\vartheta^{\prime}(s)^{2}, \\
& Q_{3}^{\vartheta}=2 \vartheta^{\prime}(s) \partial_{s}+\vartheta^{\prime \prime}(s), \\
& Q_{4}^{\vartheta}=-\partial_{s}^{2}+c_{3} \tau^{3} \kappa(s)^{3}+\tau^{3}(\kappa(s))^{4} \partial_{\tau}, \text { etc. }
\end{aligned}
$$

To finish the proof of Proposition 4.3, we solve the equation

$$
\left(\mathfrak{P}_{\varepsilon, r}^{\vartheta}-\mu\right)\left(\sum_{\ell \geq 0} a_{\ell}(s, \tau) \varepsilon^{\ell / 2}\right) \sim 0
$$

by rearranging all the terms in 4.13 in the form of a power series in $\varepsilon^{j / 2}$ and select $\vartheta, a_{\ell}(s, \tau)$ and $\mu_{\ell}$ by expressing the cancellation of each term of the formal series. We omit the details and refer to [4, 9].

Remark 4.4. The series for $\mu$ in Proposition 4.3 is the Taylor series of the first eigenvalue $\mu_{1}^{\mathrm{sw}}(\varepsilon)$. This follows from the spectral theorem.

4.4. Approximation of ground states. We recall the notation introduced in (4.3). In the sequel, we denote by

- $B_{r}(a)=\left\{s \in \mathbb{R}:\left|s-s_{r}\right| \leq a\right\}$

- $\mathfrak{v}(s)=\kappa(s)-\kappa_{\min }$.

Recall the geometric constant $\eta_{*}$ introduced in 4.1 . The following proposition is a consequence of (3.1) via the same arguments in [9, Prop. 4.7].

Proposition 4.5. (Tangential Agmon estimates) Given $\eta \in\left(0, \eta_{*}\right)$ and $0<C_{0}<M_{0} / 2$. There exist constants $c, C, R_{0}, \varepsilon_{0}>0$ such that, for all $\varepsilon \in\left(0, \varepsilon_{0}\right)$, the following is true. If $\Phi$ is a Lipschitzian function satisfying

- $\forall s \in I_{\eta, r}, \mathfrak{v}(s)-\left|\Phi^{\prime}(s)\right|^{2} \geq 0$;

- $\forall s \in I_{\eta, r} \backslash B_{r}\left(R_{0} \varepsilon^{1 / 4}\right), \mathfrak{v}(s)-\left|\Phi^{\prime}(s)\right|^{2} \geq M_{0} \varepsilon^{1 / 2}$;

- $\forall s \in B_{r}\left(R_{0} \varepsilon^{1 / 4}\right),|\Phi(s)| \leq M_{0} \varepsilon^{1 / 2} ;$

then, the following inequalities hold

$$
c \varepsilon^{3 / 2}\left\|e^{\Phi / \sqrt{\varepsilon}} u\right\|_{L^{2}\left(U_{\eta, r}\right)} \leq C\left\|e^{\Phi / \sqrt{\varepsilon}}\left(\mathfrak{P}_{\varepsilon, r}-z\right) u\right\|_{L^{2}\left(U_{\eta, r}\right)}+C \varepsilon^{3 / 2}\|u\|_{L^{2}\left(U_{\eta, r} \cap B_{r}\left(R_{0} \varepsilon^{1 / 4}\right)\right)},
$$


and

$$
\begin{aligned}
& \varepsilon^{2}\left\|\partial_{s}\left(e^{\Phi / \sqrt{\varepsilon}} u\right)\right\|_{L^{2}\left(U_{\eta, r}\right)}^{2} \\
& \quad \leq C \varepsilon^{-3 / 2}\left\|e^{\Phi / \sqrt{\varepsilon}}\left(\mathfrak{P}_{\varepsilon, r}-z\right) u\right\|_{L^{2}\left(U_{\eta, r}\right)}+C \varepsilon^{3 / 2}\|u\|_{L^{2}\left(U_{\eta, r} \cap B_{r}\left(R_{0} \varepsilon^{1 / 4}\right)\right)},
\end{aligned}
$$

for all $u \in \mathfrak{P}_{\varepsilon, r}$ and $z \in\left[\left(\frac{\pi}{2}\right)^{2}+\varepsilon \kappa_{\min },\left(\frac{\pi}{2}\right)^{2}+\varepsilon \kappa_{\min }+C_{0} \varepsilon^{3 / 2}\right]$.

Now we will use Proposition 4.5 with the following choice of $u$ and $\Phi$. First we choose $u$ as follows

where

$$
u=\psi_{\varepsilon, r}-\Pi_{r} \psi_{\varepsilon, r},
$$

- $\psi_{\varepsilon, r}(s, \tau)=\chi_{\eta, r} \Psi_{\varepsilon, \tau}(s, \tau)$;

- $\chi_{\eta, r}$ is a cut-off function supported in $I_{\eta, r}$ and such that $\chi_{\eta}=1$ on $I_{2 \eta, r}$;

- $\Psi_{\varepsilon, r}$ is the WKB solution introduced in 4.9);

- $\Pi_{r}$ is the orthogonal projection on the first eigenspace of the operator $\mathfrak{P}_{\varepsilon, r}$.

We choose $\Phi$ as follows

$$
\hat{\Phi}_{r, \eta, N, \varepsilon}(s)=\min \left\{\tilde{\Phi}_{r, N, \varepsilon}(s), \sqrt{1-\alpha} \inf _{\sigma \in I_{2 \eta, r} \backslash I_{\eta, r}}\left(\Phi_{r}(\sigma)+\int_{[s, \sigma]} \sqrt{\mathfrak{v}(\tilde{\sigma})} d \tilde{\sigma}\right)\right\},
$$

where

$$
\tilde{\Phi}_{r, N, \varepsilon}(s)=\Phi_{r}(s)-N \sqrt{\varepsilon} \max \left(\frac{\Phi_{r}}{\sqrt{\varepsilon}}, N\right) .
$$

Here $N \in \mathbb{N}, 0<\alpha<1$ and $\Phi_{r}$ is the potential introduced in 4.11. In this way, Proposition 4.5 yields the following WKB approximation (see [9, Prop. 5.1] for details).

Proposition 4.6. Let $K \subset I_{2 \eta, r}$ be a compat set. The following estimate

$$
e^{\Phi_{r} / \sqrt{\varepsilon}}\left(\Psi_{\varepsilon, r}-\Pi_{r} \Psi_{\varepsilon, r}\right)=\mathcal{O}\left(\varepsilon^{\infty}\right),
$$

holds in $\mathcal{C}^{1}\left(K ; L^{2}(0,1)\right)$.

\section{THE INTERACTION MATRIX}

5.1. Localization of the spectrum. We will use the following notation introduced by HelfferSjöstrand in [11]. Given $M>0$, by writing $r(h, \eta)=\tilde{\mathcal{O}}\left(e^{-M / h}\right)$ we mean that

- $r(h, \eta)$ is defined on a set of the form $\left(0, h_{0}\right) \times\left(0, \eta_{0}\right)$;

- There exists a function $\gamma:(0, \infty) \rightarrow \mathbb{R}$ such that $\lim _{\eta \rightarrow 0} \gamma(\eta)=0$, and for all $\alpha>0$, $h \in\left(0, h_{0}\right)$ and $\eta \in\left(0, \eta_{0}\right), r(h, \eta)=\mathcal{O}\left(e^{(\alpha+\gamma(\eta)-M) / h}\right)$.

In the sequel, we choose an arbitrary $\eta \in\left(0, \eta_{*}\right)$ where $\eta_{*}$ is the geometric constant introduced in (4.1). Some computations produce many error terms dependent on $\eta$. $\eta$ is chosen sufficiently small so that these error terms are of lower order compared to the leading terms.

Recall that $\mu_{1}^{\mathrm{sw}}(\varepsilon)$ is the ground state energy of the operators $\mathcal{P}_{\varepsilon, r}$ and $\mathcal{P}_{\varepsilon, \ell}$ and it depends on $\eta$ (see 44.2)-(4.5)). It results from the Agmon estimates in Propositions 3.1 and 4.2 , and the min-max principle that

$$
\mu_{1}^{\mathrm{sw}}(\varepsilon)-\tilde{\mathcal{O}}\left(e^{-\mathrm{S} / \sqrt{\varepsilon}}\right) \leq \lambda_{1}(\varepsilon) \leq \lambda_{2}(\varepsilon) \leq \mu_{1}^{\mathrm{Sw}}(\varepsilon)+\tilde{\mathcal{O}}\left(e^{-\mathrm{S} / \sqrt{\varepsilon}}\right) .
$$

5.2. The quasimodes. In this section, we recall the main lines of the strategy to reduce the asymptotic study of the spectral gap $\lambda_{2}\left(\mathcal{P}_{\varepsilon}\right)-\lambda_{1}\left(\mathcal{P}_{\varepsilon}\right)$ to the study of the two by two interaction matrix. To construct this matrix, we will use the groundstates of the one well problems and use them to provide an approximate basis of the space

$$
E=\bigoplus_{i=1}^{2} \operatorname{Ker}\left(\mathcal{P}_{\varepsilon}-\lambda_{i}\left(\mathcal{P}_{\varepsilon}\right)\right)
$$

We will truncate them, project them on $E$ and orthonormalize them. 
5.2.1. Truncation. Let $\chi_{\eta, r}$ (respectively $\chi_{\eta, \ell}$ ) be a cut-off function satisfying $\chi_{\eta, r}=1$ in $\{\mid s-$ $\left.s_{\ell} \mid \geq 2 \eta\right\}$ (respectively $\chi_{\eta, \ell}=1$ in $\left\{\left|s-s_{r}\right| \geq 2 \eta\right\}$ ) and $\chi_{\eta, r}=0$ in $\left\{\left|s-s_{\ell}\right| \leq \eta\right\}$ (respectively $\chi_{\eta, \ell}=0$ in $\left.\{|s-r| \leq \eta\}\right)$.

We define, for $\alpha \in\{\ell, r\}$,

$$
f_{\varepsilon, \alpha}=\chi_{\eta, \alpha} \phi_{\varepsilon, \alpha}
$$

where $\phi_{\varepsilon, \alpha}$ is the normalized ground state of the one well operator $\mathcal{P}_{\varepsilon, \alpha}$, see 4.5 and 4.2.

Thanks to the Agmon estimates in Proposition 3.3, the set $\left\{f_{\varepsilon, \ell}, f_{\varepsilon, r}\right\}$ is quasi-orthonormal in the sense that

$$
\left\|f_{\varepsilon, \alpha}\right\|^{2}=1+\tilde{\mathcal{O}}\left(e^{-2 S / \sqrt{\varepsilon}}\right) \quad \text { and } \quad\left\langle f_{\varepsilon, \alpha}, f_{\varepsilon, \beta}\right\rangle=\tilde{\mathcal{O}}\left(e^{-\mathrm{S} / \sqrt{\varepsilon}}\right) \text { for } \alpha \neq \beta .
$$

Furthermore, the function $r_{\varepsilon, \alpha}=\left(\mathcal{P}_{\varepsilon}-\mu^{\mathrm{sw}}(\varepsilon)\right) f_{\varepsilon, \alpha}, \alpha \in\{\ell, r\}$, satisfies,

$$
\left\|r_{\varepsilon, \alpha}\right\|=\tilde{\mathcal{O}}\left(e^{-\mathrm{S} / \sqrt{\varepsilon}}\right) \text {. }
$$

5.2.2. Projection. Now, we consider the new quasimodes, for $\alpha \in\{\ell, r\}$,

$$
g_{\varepsilon, \alpha}=\Pi f_{\varepsilon, \alpha},
$$

where $\Pi$ is the orthogonal projection on $E$. Thanks to (3.3), the following estimate holds, for $\alpha \in\{\ell, r\}$,

$$
\left\|g_{\varepsilon, \alpha}-f_{\varepsilon, \alpha}\right\|+\left\|\partial_{s}\left(g_{\varepsilon, \alpha}-f_{\varepsilon, \alpha}\right)\right\|=\tilde{\mathcal{O}}\left(e^{-\mathrm{S} / \sqrt{\varepsilon}}\right) .
$$

5.2.3. Orthonormalization. Starting from the basis $\left\{g_{\varepsilon, \ell}, g_{\varepsilon, r}\right\}$, we obtain by the Gram-Schmidt algorithm the orthonormal basis $\left\{\tilde{g}_{\varepsilon, \ell}, \tilde{g}_{\varepsilon, r}\right\}$. It is such that, for $\alpha \in\{\ell, r\}$,

$$
\left\|\tilde{g}_{\varepsilon, \alpha}-g_{\varepsilon, \alpha}\right\|+\left\|\partial_{s}\left(\tilde{g}_{\varepsilon, \alpha}-g_{\varepsilon, \alpha}\right)\right\|=\tilde{\mathcal{O}}\left(e^{-\mathrm{S} / \sqrt{\varepsilon}}\right) .
$$

Define $\mathrm{M}$ as the matrix of $\mathcal{P}_{\varepsilon}$ in the basis $\left\{\tilde{g}_{\varepsilon, \ell}, \tilde{g}_{\varepsilon, r}\right\}$. We have

$$
\operatorname{Spec}(\mathrm{M})=\left\{\lambda_{1}\left(\mathcal{P}_{\varepsilon}\right), \lambda_{2}\left(\mathcal{P}_{\varepsilon}\right)\right\}
$$

and, by solving the equation $\operatorname{det}(\mathrm{M}-\lambda \mathrm{I})=0$, we deduce that

$$
\lambda_{2}\left(\mathcal{P}_{\varepsilon}\right)-\lambda_{1}\left(\mathcal{P}_{\varepsilon}\right)=2\left|w_{\ell, r}\right|+\tilde{\mathcal{O}}\left(e^{-2 S / \sqrt{\varepsilon}}\right), \quad w_{\ell, r}=\left\langle r_{\varepsilon, \ell}, f_{\varepsilon, r}\right\rangle .
$$

5.3. Computation of the interaction. We may estimate the interaction term as follows. We have

$$
w_{\ell, r}=\left\langle\left(\mathcal{P}_{\varepsilon}-\mu_{1}^{\mathrm{sw}}(\varepsilon)\right) f_{\varepsilon, \ell}, f_{\varepsilon, r}\right\rangle=\left\langle\left[\mathcal{P}_{\varepsilon}, \chi_{\eta, \ell}\right] \phi_{\varepsilon, \ell}, \chi_{\eta, r} \phi_{\varepsilon, r}\right\rangle .
$$

We recall (2.9) and that $\chi_{\eta, r}$ does not depend on $\tau$. Thus,

$$
w_{\ell, r}=\left\langle\left[-\varepsilon^{2} a_{\varepsilon}^{-1}\left(\partial_{s}-i \mathrm{f}_{0}\right)\left(a_{\varepsilon}^{-1}\left(\partial_{s}-i \mathrm{f}_{0}\right)\right), \chi_{\eta, \ell}\right] \phi_{\varepsilon, \ell}, \chi_{\eta, r} \phi_{\varepsilon, r}\right\rangle .
$$

The explicit expression of the commutator and an integration by parts yield

$$
w_{\ell, r}=\varepsilon^{2} \int_{(-L, L) \times(0,1)} a_{\varepsilon}^{-1} \chi_{\eta, \ell}^{\prime} \chi_{\eta, r}\left(\overline{\left(\partial_{s}-i \mathrm{f}_{0}\right) \phi_{\varepsilon, r}} \phi_{\varepsilon, \ell}-\left(\partial_{s}-i \mathrm{f}_{0}\right) \phi_{\varepsilon, \ell} \overline{\phi_{\varepsilon, r}}\right) d s d t
$$

so that, by support consideration $\left(\chi_{\eta, r}=1\right.$ on $\left.\operatorname{supp} \chi_{\eta, \ell}\right)$,

$$
w_{\ell, r}=\varepsilon^{2} \int_{(-L, L) \times(0,1)} a_{\varepsilon}^{-1} \chi_{\eta, \ell}^{\prime}\left(\overline{\left(\partial_{s}-i \mathrm{f}_{0}\right) \phi_{\varepsilon, r}} \phi_{\varepsilon, \ell}-\left(\partial_{s}-i \mathrm{f}_{0}\right) \phi_{\varepsilon, \ell} \overline{\phi_{\varepsilon, r}}\right) d s d t .
$$

Note that $\chi_{\eta, \ell}^{\prime}$ is supported in $(-L, 0)$. After another integration by parts, we get

$$
\begin{aligned}
w_{r, \ell}= & \tilde{w}_{r, \ell}+\varepsilon^{2} \int_{(0,1)} a_{\varepsilon}^{-1}\left(\overline{\left(\partial_{s}-i \mathrm{f}_{0}\right) \phi_{\varepsilon, r}} \phi_{\varepsilon, \ell}-\left(\partial_{s}-i \mathrm{f}_{0}\right) \phi_{\varepsilon, \ell} \overline{\phi_{\varepsilon, r}}\right)(0, t) d t \\
& -\varepsilon^{2} \int_{(0,1)} a_{\varepsilon}^{-1}\left(\overline{\left(\partial_{s}-i \mathrm{f}_{0}\right) \phi_{\varepsilon, r}} \phi_{\varepsilon, \ell}-\left(\partial_{s}-i \mathrm{f}_{0}\right) \phi_{\varepsilon, \ell} \overline{\phi_{\varepsilon, r}}\right)(-L, t) d t
\end{aligned}
$$


where

$$
\begin{aligned}
\tilde{w}_{r, \ell}= & -\varepsilon^{2} \int_{(-L, 0) \times(0,1)} \chi_{\eta, \ell} \partial_{s}\left(a_{\varepsilon}^{-1} \overline{\left(\partial_{s}-i \mathrm{f}_{0}\right) \phi_{\varepsilon, r}} \phi_{\varepsilon, \ell}\right) d s d t \\
& +\varepsilon^{2} \int_{(-L, 0) \times(0,1)} \chi_{\eta, \ell} \partial_{s}\left(a_{\varepsilon}^{-1}\left(\partial_{s}-i \mathrm{f}_{0}\right) \phi_{\varepsilon, \ell} \overline{\phi_{\varepsilon, r}}\right) d s d t .
\end{aligned}
$$

Let us explain why $\tilde{w}_{r, \ell}=0$. We have to deal with the flux in this last expression. Letting $\tilde{\phi}_{\varepsilon, \alpha}=e^{-i f_{0} s} \phi_{\varepsilon, \alpha}$, we have

$\tilde{w}_{r, \ell}=-\varepsilon^{2} \int_{(-L, 0) \times(0,1)} \chi_{\eta, \ell} \partial_{s}\left(a_{\varepsilon}^{-1} \overline{\partial_{s} \tilde{\phi}_{\varepsilon, r}} \tilde{\phi}_{\varepsilon, \ell}\right) d s d t+\varepsilon^{2} \int_{(-L, 0) \times(0,1)} \chi_{\eta, \ell} \partial_{s}\left(a_{\varepsilon}^{-1} \partial_{s} \tilde{\phi}_{\varepsilon, \ell} \overline{\tilde{\phi}_{\varepsilon, r}}\right) d s d t$,

and thus

$\tilde{w}_{r, \ell}=-\varepsilon^{2} \int_{(-L, 0) \times(0,1)} \chi_{\eta, \ell} \partial_{s}\left(a_{\varepsilon}^{-1} \overline{\partial_{s} \tilde{\phi}_{\varepsilon, r}}\right) \tilde{\phi}_{\varepsilon, \ell} d s d t+\varepsilon^{2} \int_{(-L, 0) \times(0,1)} \chi_{\eta, \ell} \partial_{s}\left(a_{\varepsilon}^{-1} \partial_{s} \tilde{\phi}_{\varepsilon, \ell}\right) \overline{\tilde{\phi}_{\varepsilon, r}} d s d t$,

so that

$$
\begin{aligned}
\tilde{w}_{r, \ell}= & -\varepsilon^{2} \int_{(-L, 0) \times(0,1)} \chi_{\eta, \ell} \overline{\left(\partial_{s}-i \mathrm{f}_{0}\right)\left(a_{\varepsilon}^{-1}\left(\partial_{s}-i \mathrm{f}_{0}\right) \phi_{\varepsilon, r}\right)} \phi_{\varepsilon, \ell} d s d t \\
& +\varepsilon^{2} \int_{(-L, 0) \times(0,1)} \chi_{\eta, \ell}\left(\partial_{s}-i \mathrm{f}_{0}\right)\left(a_{\varepsilon}^{-1}\left(\partial_{s}-i \mathrm{f}_{0}\right) \phi_{\varepsilon, \ell}\right) \overline{\phi_{\varepsilon, r}} d s d t .
\end{aligned}
$$

Then, it remains to notice that $\phi_{\varepsilon, \alpha}$ satisfies the eigenvalue equation and we get that $\tilde{w}_{r, \ell}=0$.

Thus,

$$
\begin{aligned}
w_{r, \ell}= & \varepsilon^{2} \int_{(0,1)} a_{\varepsilon}^{-1}\left(\overline{\left(\partial_{s}-i \mathrm{f}_{0}\right) \phi_{\varepsilon, r}} \phi_{\varepsilon, \ell}-\left(\partial_{s}-i \mathrm{f}_{0}\right) \phi_{\varepsilon, \ell} \overline{\phi_{\varepsilon, r}}\right)(0, t) d t \\
& -\varepsilon^{2} \int_{(0,1)} a_{\varepsilon}^{-1}\left(\overline{\left(\partial_{s}-i \mathrm{f}_{0}\right) \phi_{\varepsilon, r}} \phi_{\varepsilon, \ell}-\left(\partial_{s}-i \mathrm{f}_{0}\right) \phi_{\varepsilon, \ell} \overline{\phi_{\varepsilon, r}}\right)(-L, t) d t .
\end{aligned}
$$

We can now use the WKB approximation of Proposition 4.6 and the symmetry relation between $\phi_{\varepsilon, \ell}$ and $\phi_{\varepsilon, r}$. We also notice that $a_{\varepsilon}=1+\mathcal{O}(\varepsilon)$. Then, by separation of variables, we are reduced to the interaction term of the effective model $\varepsilon^{2} \mathcal{L}_{\varepsilon}^{\text {eff }}$ (with respect to $s$, the WKB Ansatz is exactly the one of the effective operator). We refer to [4, Section 4] where this (one dimensional) interaction is explicitly computed. The phase factor of the last two integrals can be computed with (4.7): the first integral (up contribution) is real and the phase factor of the second one (down contribution) is $e^{-2 i L f_{0}}$. To get the tunneling estimate for the initial operator $\mathcal{L}_{\varepsilon}$ (or $\widetilde{\mathcal{L}}_{\varepsilon}$, see (2.8), it remains to divide by $\varepsilon^{2}$.

Acknowledgments. This work was initiated when the authors visited the Banff International Research Station (workshop 17w5110 "Phase transition models").

\section{REFERENCES}

[1] V. Bonnaillie-Noël and M. Dauge. Asymptotics for the low-lying eigenstates of the Schrödinger operator with magnetic field near corners. Ann. Henri Poincaré, 7(5):899-931, 2006.

[2] V. Bonnaillie-Noël, F. Hérau, and N. Raymond. Curvature induced magnetic bound states: towards the tunneling effect for the ellipse. Journées équations aux dérivées partielles, Exp. 3, 2016.

[3] V. Bonnaillie-Noël, F. Hérau, and N. Raymond. Magnetic WKB constructions. Arch. Ration. Mech. Anal., 221(2):817-891, 2016.

[4] V. Bonnaillie-Noël, F. Hérau, and N. Raymond. Semiclassical tunneling and magnetic flux effects on the circle. J. Spectr. Theor. (in press), 2017.

[5] P. Duclos and P. Exner. Curvature-induced bound states in quantum waveguides in two and three dimensions. Rev. Math. Phys., 7(1):73-102, 1995.

[6] S. Fournais and B. Helffer. Spectral methods in surface superconductivity, volume 77 of Progress in Nonlinear Differential Equations and their Applications. Birkhäuser Boston, Inc., Boston, MA, 2010.

[7] R. L. Frank. On the tunneling effect for magnetic Schrödinger operators in antidot lattices. Asymptot. Anal., 48(1-2):91-120, 2006. 
[8] B. Helffer and A. Kachmar. Eigenvalues for the Robin Laplacian in domains with variable curvature. Trans. Amer. Math. Soc., 369(5):3253-3287, 2017.

[9] B. Helffer, A. Kachmar, and N. Raymond. Tunneling for the Robin Laplacian in smooth planar domains. Commun. Contemp. Math., 19(1):1650030, 38, 2017.

[10] B. Helffer and K. Pankrashkin. Tunneling between corners for Robin Laplacians. J. Lond. Math. Soc. (2), 91(1):225-248, 2015.

[11] B. Helffer and J. Sjöstrand. Multiple wells in the semiclassical limit. I. Comm. Partial Differential Equations, 9(4):337-408, 1984.

[12] D. Krejčiřík. Spectrum of the Laplacian in a narrow curved strip with combined Dirichlet and Neumann boundary conditions. ESAIM Control Optim. Calc. Var., 15(3):555-568, 2009.

[13] D. Krejčiřík, N. Raymond, and M. Tušek. The magnetic Laplacian in shrinking tubular neighborhoods of hypersurfaces. J. Geom. Anal., 25(4):2546-2564, 2015.

[14] A. Outassourt. Comportement semi-classique pour l'opérateur de Schrödinger à potentiel périodique. $J$. Funct. Anal., 72(1):65-93, 1987.

[15] K. Pankrashkin and N. Popoff. An effective hamiltonian for the eigenvalue asymptotics of the robin laplacian with a large parameter. J. Math. Pures Appl., 106(4):615-650, 2016.

(A. Kachmar) Lebanese University, Department of Mathematics, Nabatiyeh, Lebanon.

E-mail address: ayman.kashmar@gmail.com

(N. Raymond) IRMaR - UMR6625, Université Rennes 1, CNRS, Campus de Beaulieu, F-35042 Rennes Cedex, France

E-mail address: nicolas.raymond@univ-rennes1.fr 